\title{
A systematic review of the methods used to assess fluid intake in working adults: the fluid at work study
}

\author{
G. Rogerson ${ }^{1}$, M. Whelan ${ }^{1,2}$ and R. Gibson ${ }^{1}$ \\ ${ }^{1}$ Department of Nutritional Sciences, King's College London, London, UK and \\ ${ }^{2}$ British Dietetic Association Work Ready, Interchange Place, Birmingham, UK
}

Adequate fluid intake is essential for human physical health and mental function. Mild dehydration may be detrimental to physical, psychomotor, and cognitive performance resulting in degraded mood, increased perception of task difficulty and reduced ability to concentrate ${ }^{(1,2,3)}$. Assessing fluid intake in the UK workforce is challenging due to the lack of a valid and reliable measurement tool ${ }^{(4)}$. This review aimed to 1) identify and characterise existing fluid intake measurement tools used in the workplace setting or amongst free-living, healthy adults of working age and 2) report current validation status of available assessment tools. PROSPERO registration CRD42021221288.

The systematic review was conducted in line with The Preferred Reporting Items for Systematic reviews and Meta-Analyses (PRISMA). Electronic databases EMBASE, PUBMED and SCOPUS to June 2021 were searched to identify relevant studies measuring fluid intake using a defined tool or method. Search terms were generated using terms for 'workplace' and 'fluid/beverage intake'.

Additional studies were identified by hand from trial registers, grey literature, and reference lists. Eligibility was determined using pre-defined inclusion and exclusion criteria. Studies were included if they measured fluid intake using a defined method or tool; were conducted in free-living adults aged 18-65 years, or in a specific workplace; tested a fluid intake intervention and were published in English since 1990. Two authors (GR and RG) screened all titles and abstracts. Full text articles were retrieved if abstracts appeared to meet the inclusion criteria. Where any disagreement occurred regarding inclusion, a third author assessed suitability (MW). Study quality was assessed using a modified STROBE (Strengthening the Reporting of Observational Studies in Epidemiology) checklist ${ }^{(5)}$. Narrative synthesis was performed and results thematically analysed.

The review identified 99 eligible studies, 3 of which were conducted in a UK population. Occupational populations included healthcare workers $(n=11)$, university communities $(n=10)$, miners $(n=4)$, construction workers $(n=3)$, airport workers $(n=3)$, agricultural workers $(n=3)$, firefighters $(n=3)$, police officers $(n=1)$. Food intake diaries and $24 \mathrm{hr}$ recalls were commonly used dietary assessment methods, reported in 33 and 12 studies respectively. Food frequency or screening questionnaires were used in 38 studies, of which 7 were identified as specific fluid/beverage assessment tools. None of the specific fluid/beverage assessment tools were validated in a UK population. Five tools reported a relative validity score $>0.4$ for total daily fluid intake.

The fluid/beverage measurement tools identified in this review will inform the development of a new fluid intake assessment tool, which will be taken forward for evaluation in the UK working population as part of the Fluid at Work study.

\section{Acknowledgments}

The Fluid at Work study is funded by a British Dietetic Association GET award grant (20/11).

\section{References}

1. Armstrong LE, Ganio MS et al. (2012) J Nutr 142, 382-388.

2. Wilson MM \& Morley JE (2003) Eur J Clin Nutr 57, S24-29.

3. Shirreffs SM, Merson SJ, et al. (2004) Br J Nutr 91, 951-958.

4. Gibson S, Gunn P \& Maughan RJ (2012) Nutr Bull 37, 182-192.

5. Lachat, C, Hawwash D, et al. (2016) Nutr Bull 41, 240-251. 